\title{
Photovoltaic tweezers an emergent tool for applications in nano and bio-technology
}

\author{
M. Carrascosa ${ }^{\mathrm{a}}$, A. García-Cabañes ${ }^{\mathrm{a}}$, M. Jubera, I. Elvira ${ }^{\mathrm{a}}$, H. Burgos ${ }^{\mathrm{a}}$, J. L. Bella ${ }^{\mathrm{b}}$, F. Agulló- \\ López $^{c}$, J. F. Muñoz-Martínez ${ }^{\mathrm{a}, \mathrm{d}}$, A. Alcazar ${ }^{\mathrm{d}}$ \\ ${ }^{a}$ Departamento de Física de Materiales, Universidad Autónoma de Madrid, Madrid 28049, Spain. \\ ${ }^{\mathrm{b}}$ Departamento de Biología, Universidad Autónoma de Madrid, Madrid 28049, Spain. \\ ${ }^{c}$ Centro de Microanálisis de Materiales, Universidad Autónoma de Madrid, Madrid 28049, Spain. \\ ${ }^{\mathrm{d}}$ Departamento de Mecánica de Fluidos y Propulsión Aeroespacial, Universidad Politécnica de \\ Madrid, Madrid 8040, Spain.
}

\begin{abstract}
An overview of the work recently conducted by our group on the development and applications of photovoltaic tweezers is presented. It includes the analysis of the physical basis of the method and the main achievements in its experimental implementation. Particular attention will be paid to the main potential applications and first demonstrations of its use in nano- and bio-technology. Specifically: $i$ ) fabrication of metallic nanoestructures for plasmonic applications, ii) development of diffractive components, iii) manipulation and patterning (1D and 2D) of various types of bio-objects (spores or pollen...) and $i v$ ) effects of PV fields of $\mathrm{LiNbO}_{3}$ in tumour cells.
\end{abstract}

Keywords: Optical tweezers, photovoltaic tweezers, bulk photovoltaic effect, photorefractive $\mathrm{LiNbO}_{3}$, plasmonic structures, bio-object patterning.

\section{INTRODUCTION}

The manipulation of micro- and nano-objects, such as molecules, living cells or bacteria and micro- and nanoparticles, is a subject of increasing importance for a variety of applications in nano-technology, photonics, biotechnology and biomedicine. A number of different approaches have been proposed and explored including conventional optical tweezers or electrokinetical methods. In the last years a new optoelectric technique for particle manipulation, the so called photovoltaic (PV) tweezers (or photorefractive tweezers), has been proposed and demonstrated [1-3]. It relies in the bulk photovoltaic effect (PVE) [4], a singular phenomenon which appears in some crystalline ferroelectric materials (doped $\mathrm{LiNbO}_{3}$ clearly highlighting) when properly doped (mainly with $\mathrm{Fe}$ ). It allows the photo-generation of very high internal electric fields $\left(1-3 \times 10^{5} \mathrm{~V} / \mathrm{cm}\right)$ for moderate or low light levels $\left(\sim \mathrm{mW} / \mathrm{cm}^{2}\right)[4-5]$. The field extends to the proximity of the crystal surface (evanescent field) and can trap either charged particles via electrophoretic (coulombian) forces or neutral particles via dielectrophoretic forces. In fact, either dielectric or metallic particles have been manipulated $[3,6,7]$.

The novel technique has a number of key advantages over conventional optical tweezers and/or electro-kinetical methods such as parallel manipulation on many particles, flexibility and versatility of the light-induced patterning without the need of electrodes or voltage suppliers, operation at low light intensities (several orders of magnitude smaller than conventional optical tweezers) and reconfiguration capabilities [8]. However, in spite of its potential, the technique is still in an emergent stage and its possibilities and, particularly, its potential applications are in continuous expansion.

In this contribution we present an overview of the work recently conducted by our group on the development and applications of PV tweezers. It includes a brief analysis of the physical basis of the method and the main achievements in its experimental implementation in section 2 and 3 respectively. Particular attention will be paid in section 4 to our

*m.carrascosa@uam.es; phone 34 914973814; fax 34 914978579; 
recent new results on various possible applications in nano- and bio-technology. Specifically: $i$ ) fabrication of metallic nanoestructures for plasmonic applications, $i$ ) development of diffractive components, iii) manipulation and patterning (1D and 2D) of various types of bio-objects (spores or pollen...) and $i v$ ) effects of PV fields of $\mathrm{LiNbO}_{3}$ in tumour cells.

\section{PHYSICAL BASIS OF PV TWEEZERS}

The physical effect accounting for the evanescent fields responsible for the trapping and patterning of nano-particles is the PV effect in $\mathrm{Fe}-\mathrm{LiNbO}_{3}$. Suitable light illumination induces an asymmetric excitation of electrons from the Fe ${ }^{2+}$ donor centers along the ferroelectric trigonal (poling) axis that abruptly generates an electrical dipole moment, $p_{P V}=e l_{P V}$, $l_{P V}$ being the PV drift length $(\sim 1-5 \AA)$. A PV electric current is induced,

$$
j_{P V}=e \alpha l_{P V} I
$$

( $I$ being the light intensity and $\alpha$ the absorption coefficient), followed by charge migration and redistribution due to electron re-trapping at acceptors $\mathrm{Fe}^{3+}$ centers. Then, a correlated space-charge field is developed, both, inside and outside (evanescent field) the sample. There are two possible orientations of the substrate: $i$ ) the more conventional one in this field which has the polar PV axis parallel to surface (parallel configuration) and $i$ ) the perpendicular configuration with the polar axis normal to the surface recently used to improve 2D patterning $[9,10]$.

Experiments have been very often performed in the parallel configuration, under a periodic light intensity profile and in this case a detailed analytical analysis has been reported [7,11]. The light intensity pattern can be written

$$
I=I_{0}(1+m \cos K x)
$$

$\mathrm{Z}$ being the normal to the slab, $X$ the $\mathrm{PV}$ (poling) axis that coincides with the trigonal crystallographic axis of $\mathrm{LiNbO}_{3}$ and $m(0<m<1)$ is the modulation index of the light pattern. In accordance with the standard photorefractive model significant electric fields develop inside the sample and extend as evanescent fields into the surrounding medium. Most analyses of the effect deal with the bulk fields generated in an infinite medium, since they are mainly relevant for optical applications. On the other hand, the evanescent fields appearing at the interface between the PR crystal and the outside medium have been generally ignored. For low $m$ values a linear approximation to carrier transport equations applies. Then, under steady-state conditions, the field profiles outside the crystal $(z<0)$ differ from those in an infinite medium and consist of parallel and perpendicular components whose periodicities present a $\pi / 2$ phase mismatch [11] . After taking into account the electromagnetic boundary conditions at the sample-medium interface the evanescent fields $(z>0)$ write:

$$
\begin{gathered}
E_{X, \text { out }}(x, z)=-m E_{P V} \frac{\varepsilon}{\varepsilon_{m}+\varepsilon} e^{-K z} \cos K x \\
E_{Z, \text { out }}(x, z)=m E_{P V} \frac{\varepsilon}{\varepsilon_{m}+\varepsilon} e^{-K z} \sin K x
\end{gathered}
$$

where $E_{P V}$ is the photovoltaic field and $\varepsilon$ and $\varepsilon_{m}$ are the static dielectric constants of the PV material and surrounding medium, respectively. When the light intensity profile has a high contrast ( $m$ close to 1$)$ the space charge fields present harmonic contributions with $K$ vectors that are multiple of the fundamental $K$ of the light. The impact of this aspect on PV tweezers has been also addressed in [11] considering an approximation that keeps the fundamental and the second harmonic components. The result indicates the importance of the presence of harmonics on the trapping and patterning processes. The dielectrophoretic force acting on a neutral dielectric particle placed at the sample surface is:

$$
\vec{f}=-\nabla(-\vec{p} \cdot \vec{E})=\varepsilon_{0} \alpha_{p} \nabla\left(\vec{E}^{2}\right)
$$

$\alpha_{p}$ being the static polarizability tensor of the particle. For spherical and crystallographically isotropic particles, in the Rayleigh approximation $(R<<\Lambda=1 / K)$, the polarizability is a scalar and writes: 


$$
\alpha_{p}=\frac{3}{2} V \varepsilon_{m} \frac{\varepsilon_{p}-\varepsilon_{m}}{\varepsilon_{p}+2 \varepsilon_{m}}
$$

$V$ being the volume of the particle and $\varepsilon_{m}$ and $\varepsilon_{p}$ the dielectric permittivities of the host medium (air or liquid) and particle, respectively. More specific expressions and analysis for the dielectrophoretic force on the particles can be found in references [7,11]. In addition theoretical calculations of the operation of PV tweezers with arbitrary light patterns have been reported in [12] including successful comparison with experimental results.

\section{EXPERIMENTAL METHODS: 1D AND 2D PATTERNING}

PV tweezers allow generating very flexible 1D and 2D patterning of micro- and nanoparticles that are essentially a replica of the light distribution. As PV substrate we have used $\mathrm{LiNbO}_{3}$ highly doped with iron with two different geometries, $\mathrm{x}$ - and z-cut substrates, with the polar axis parallel and perpendicular to the crystal surface, respectively. The use of the perpendicular configuration is a key point to obtain high quality $2 \mathrm{D}$ patterns $[9,10]$

The technique for particle trapping and patterning includes two steps that can be either simultaneous or successive: $i$ ) Illumination of the PV substrate $\left(\mathrm{Fe}: \mathrm{LiNbO}_{3}\right)$ and, ii) particle deposition. The sequential procedure is easier to implement and it is the one we use ordinarily. Illumination has been carried out with different procedures depending on the application. For 1D periodic patterning we use a two-beam interferometric set-up $[7,13]$ whereas for 2D patterning the key element is spatial light modulator that allows to generate 2D light patterns that are projected on the substrate $[6,9]$. The particles or bio-objects are deposited from air or from a hexane suspension in which the substrate is immersed.

Two illustrative examples are presented in Figure 1. Figure 1a shows an image of a pattern of graphite particles (diameter $d \sim 1 \mu \mathrm{m}$ ) deposited after sinusoidal interferometric illumination with a period of $60 \mu \mathrm{m}$ on an $\mathrm{x}$-cut sample (parallel configuration). Figure 1b shows a $2 \mathrm{D}$ pattern of Al nanoparticles $(d \sim 70 \mathrm{~nm})$ obtained using a spatial light modulator and the perpendicular configuration for the substrate. In both cases particle deposition has been developed from a hexane suspension of particles.

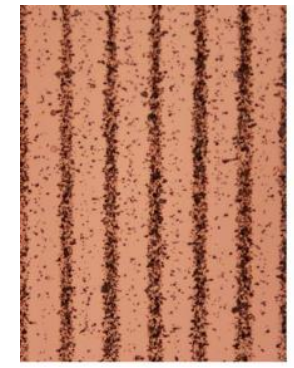

(a)

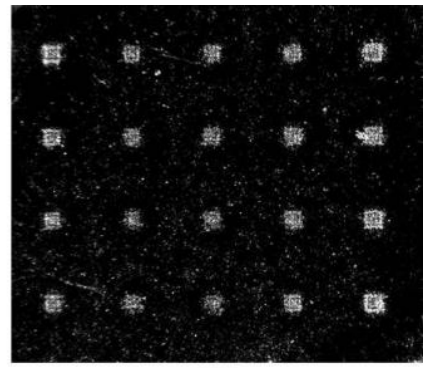

(b)

Figure 1. (a) Photograph of a graphite 1D particle pattern obtained by sinusoidal illumination of an x-cut $\mathrm{Fe}: \mathrm{LiNbO}_{3}$ substrate. The pattern period is $60 \mu \mathrm{m}$, i.e. the same periodicity of light. (b) Photograph of a $2 \mathrm{D}$ pattern of $\mathrm{Al}$ nanoparticles deposited on a z-cut Fe: $\mathrm{LiNbO}_{3}$ substrate after illumination with a light pattern of squares of $245 \mu \mathrm{m}$ side separated $1 \mathrm{~mm}$.

When light is removed, the PV fields remain during a long time. Typical times are in the range of minutes-months. However, they can be erased either by uniform illumination or by heating the substrate above RT. Therefore, one has a means to record sequentially different patterns on the same crystal surface and so, the patterning method offers reconfigurability capabilities [8]. In Figure 2 we present a transmission microscope image of a particle pattern obtained with Ag particles deposited in air by spraying from beneath the surface. The image shows a central circular region in which the PV field has been erased by homogeneous illumination provided by the microscope, whereas it is kept in the surroundings. 


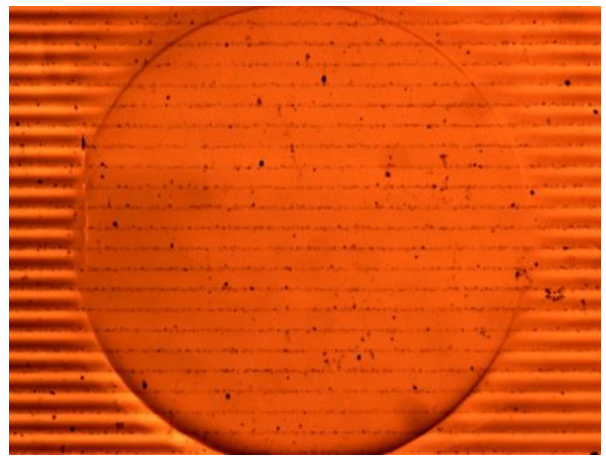

Figure 2: Transmission microphotograph of an Ag pattern deposited in air after sinusoidal illumination of the substrate with $\Lambda=50 \mu \mathrm{m}$. After particle patterning the PV field of the central circular region has been "erased" by illumination through the microscope objective.

\section{APPLICATIONS}

So far only very few real applications have been reported [9] although a large span of possibilities can be envisaged. Recently we have addressed the investigation of a number of possible applications in nano- and bio-technology that we describe below.

\subsection{Metallic nanostructures}

There is an increasing interest in producing structures of metallic nanoparticles because they have multiple applications in the fields such as photonics or bio-devices. As it has been already seen in section 3, PV tweezers are a very useful tool to fabricate metallic particle patterns. We have successfully used Ag, Au and Al nanoparticles with diameters ranging between 10 and $150 \mathrm{~nm}$. 1D and 2D patterns have been obtained. In fact, the minimum reported periods of 1D patterns obtained with PV tweezers have been fabricated by our group using Al nanoparticle with 70 nm-diameter [13]. Figure 3a shows a microscope image of a pattern of $\mathrm{Ag}(d \sim 35 \mathrm{~nm})$ nanoparticles. To have a first characterization of the plasmonic behavior of the structure its relative reflectance with regard to nude $\mathrm{Fe}: \mathrm{LiNbO}_{3}$ has been measured. The results for probe light linearly polarized parallel and perpendicular to the particle fringes are shown in Figure $3 \mathrm{~b}$. A broad, but weak band can be seen both polarizations. The peak (minimum reflectance) for the parallel polarization $(\sim 640 \mathrm{~nm})$ is clearly shifted to longer wavelengths than the one for the perpendicular polarization $(\sim 600 \mathrm{~nm})$. This result that appears in all the 1D metallic structures measured, may be justified taking into account that the geometry of structure should lead to a more effective coupling between nanoparticles in the direction parallel to the fringes.

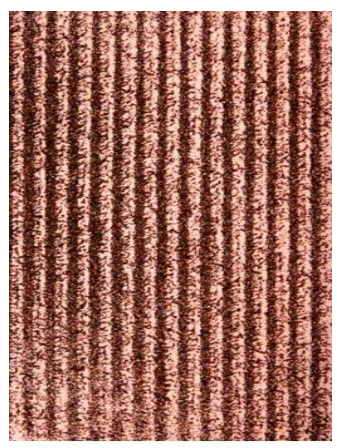

(a)

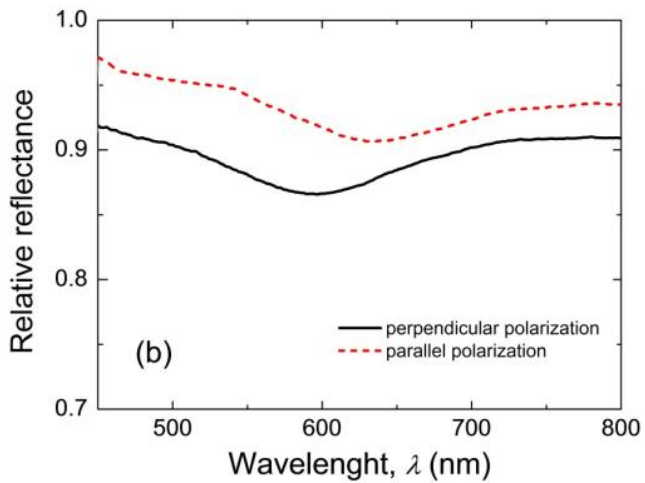

Figure 3: (a) Microscopic image of a periodic pattern $(\Lambda=20 \mu \mathrm{m})$ of Ag nanoparticles (in black) (b) Relative reflectance with regard to $\mathrm{Fe}: \mathrm{LiNbO}_{3}$ for two polarizations of the incident light, parallel (red) and perpendicular (black) to the fringes. 


\subsection{Particle patterns for diffractive components}

Diffractive optical components play a key role in a variety of photonics devices. They include diffraction gratings, beam deflectors, tuneable filters, Bragg reflectors, grating couplers, Fresnel lenses and so on. A possible photonic application of particle patterns is just to provide a simple and flexible method to fabricate diffractive structures with arbitrary periodic 1D and 2D designs with dielectric or metallic particles. A very simple example is a fringe pattern as the one illustrated that is shown in Figure 4a. It has been obtained with dielectric $\mathrm{CaCO}_{3}$ micro-particles and has 67 lines $/ \mathrm{mm}$. The corresponding diffraction diagram is shown in Figure $4 \mathrm{~b}$.
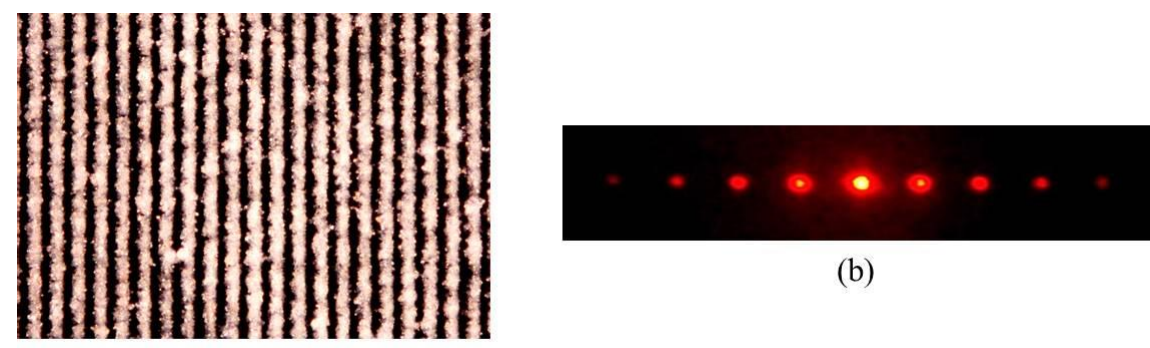

(b)

(a)

Figure 4: (a) Microscope image of a region of the diffractive structure fabricated by trapping of $\mathrm{CO}_{3} \mathrm{Ca}$ particles after sinusoidal illumination with a period $\Lambda=15 \mu \mathrm{m}$. (b) Diffractive transmission pattern of the structure.

\subsection{Trapping and patterning of bio-objects}

Once the possibility of flexible trapping and patterning of micro- and nanoparticles was demonstrated, a further step, not reported so far is to check the possibility of patterning different kind of bio-objects with PV tweezers. Very recently we have addressed a set of experiments directed to trap and pattern spores and pollen. In Figure 5 we show an illustrative result for spores with $10-\mu \mathrm{m}$ diameter (see Fig. 5a). The trapping experiment have been carried out in x-cut substrate and under sinusoidal illumination $(\Lambda=35 \mu \mathrm{m})$. The spores have been deposited immersing the substrate in a hexane suspension. Figure $5 \mathrm{~b}$ shows an image of the observed spore pattern and Figure 5c shows the result of a control experiment in which the substrate have been immersed in the hexane suspension without any previous illumination. In Figure $5 \mathrm{~b}$ a $1 \mathrm{D}$ pattern formed by lines of spores perpendicular to the polar axis is clearly seen whereas in Fig. $5 \mathrm{c}$, without PV fields, only some spores remain in the surface but located at random. The trapping experiments have been also conducted with deposition in air obtaining similar results. Therefore, a first demonstration of the capabilities of PV tweezers to pattern biological objects has been obtained.

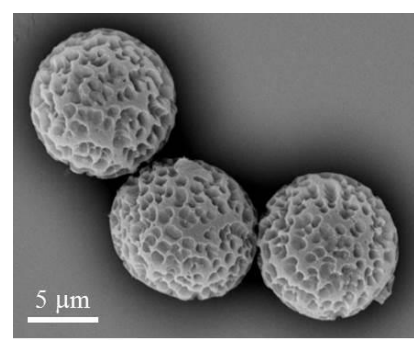

(a)

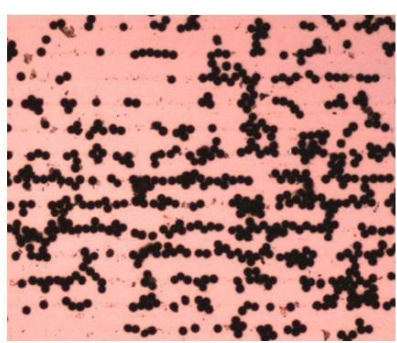

(b)

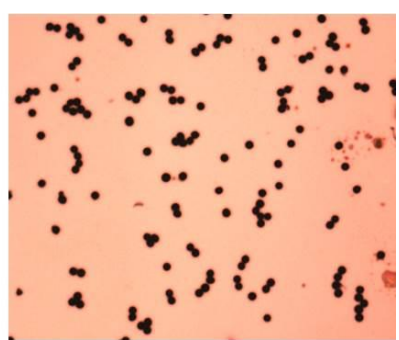

(c)

Figure 5: (a) SEM image of one spore. (b) $35 \mu \mathrm{m}$ period spores pattern obtained after sinusoidal illumination. (c) Control experiment with a non-illuminated substrate, i.e. without PV fields.

A second set of experiments were carried out using pollen grains with a diameter of $\sim 70 \mu \mathrm{m}$ (see Figure $6 \mathrm{a}$ ). Due to the large size of these pollen grains only macroscopic patterns (in the scale of $\mathrm{mm}$ ) have been obtained. However, using pollen fragments obtained by sonication high quality 1D and 2D patterns of the biological powder are obtained. This is 
shown in the microscopic images of Figures 5c and 5d, respectively. It is worthwhile mentioning that in the case of $1 \mathrm{D}$ structuring of pollen fragments periodic patterns, with spatial period in the range between 1 and $100 \mu \mathrm{m}$, have been obtained with a very good quality. In fact, the smallest period value of $1.7 \mu \mathrm{m}$ is a record for this kind of tweezers, lower than minimum periods reported so far $(4.5 \mu \mathrm{m}$ [13]) when using metallic and dielectric nanoparticles. Then, these results can be considered very promising regarding the capabilities of this kind of tweezers to trap and manipulate bio-objects and open the door to a wide number of applications in biotechnology.

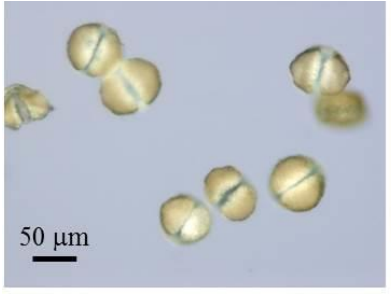

(a)

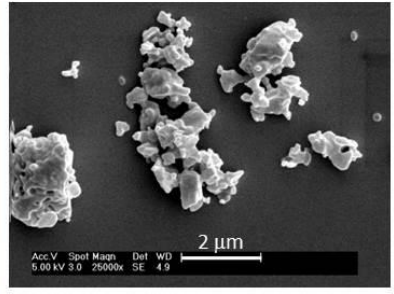

(b)

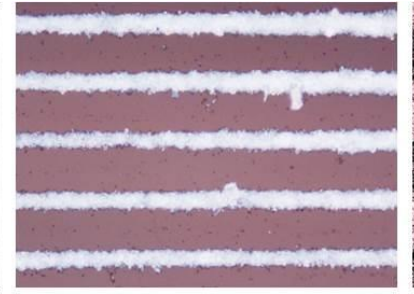

(c)

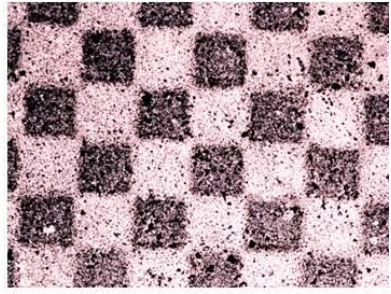

(d)

Figure 6: (a) Microscope image of pollen grains and (b) SEM image of pollen fragments. (c) Microscope image of a $65 \mu \mathrm{m}$ period pattern of pollen fragments obtained after sinusoidal illumination. (d) Microscope image of a 2D pattern of pollen fragments obtained after illumination with mosaic of squares $(100 \mu \mathrm{m}$ side $)$ provided by a spatial light modulator.

\subsection{Effects of PV fields in tumor cells}

Finally, it is worthwhile mentioning another application of the interaction between PV fields and cells. We have already demonstrated [14] that the evanescent PV field has a dramatic effect in human tumor cells (HeLa cell line).Two different kind of experiments have been performed: $i$ ) culturing the cells on a $\mathrm{Fe}: \mathrm{LiNbO}_{3}$ substrate and afterwards exposing the culture to low-intensity visible light $\left(10-100 \mathrm{~mW} / \mathrm{cm}^{2}\right)$, ii) incubating the cells with Fe:LiNbO $\mathrm{Licroparticles}_{3}(1-3 \mu \mathrm{m}$ diameter) and illuminating with $40 \mathrm{~mW} / \mathrm{cm}^{2}$ green light. In both cases necrotic death of nearly all cells occurs when the substrate is illuminated to generate the PV fields. The results are plotted in Figure 7a.

These very remarkable effects of PV fields in cells could become very relevant for therapy purposes if the size of PV particles is reduced to a nanometer scale. Preliminary experiments along this direction have been addressed [15] using Fe: $\mathrm{LiNbO}_{3}$ nanoparticles $(d \sim 100 \mathrm{~nm})$ by grinding from crystal or by bottom-up sol-gel chemical synthesis from metaethoxide precursors. The nanoparticles were incorporated into a tumor cell culture and it was subjected to 30-min illumination with a blue LED. Control experiments of the culture with nanoparticles in dark were also carried out for reference. The percent of cell death in dark and under illumination conditions as a function of the nanoparticle concentration is plotted in Figure 7b. Although the cell death fraction is larger for the illuminated cultures dark toxicity itself is high and much further work is necessary to progress in this direction.
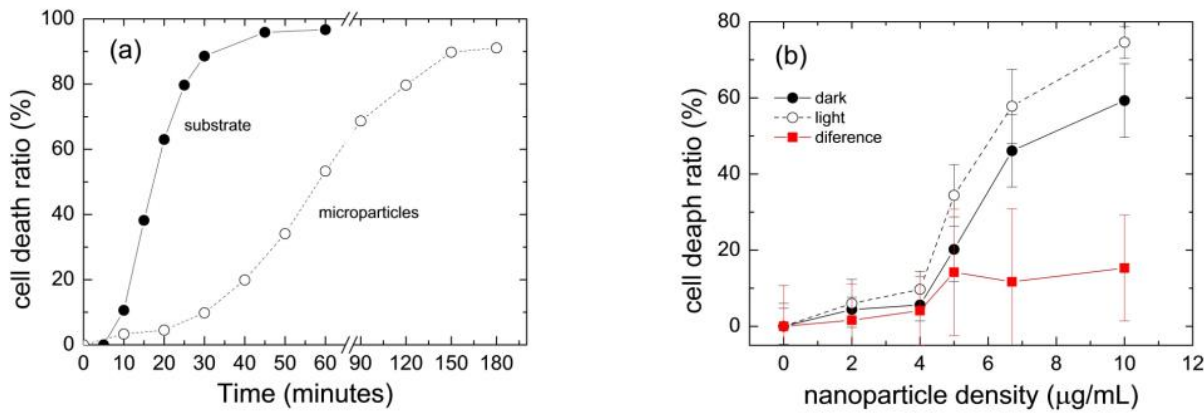

Figure 7: (a) Time evolution of the fraction of dead cells evaluated through morphological criteria for tumor cells cultured on Fe: $\mathrm{LiNbO}_{3}$ substrates (solid circles) or in contact with $\mathrm{Fe}: \mathrm{LiNbO}_{3}$ microparticles (open circles). (b) Fraction of dead cells vs $\mathrm{Fe}: \mathrm{LiNbO}_{3}$ nanoparticle density under illumination (open circles) and in dark (solid circles). The difference between both curves is also represented (red solid squares). 


\section{SUMMARY AND CONCLUSIONS}

A description of the operation of PV tweezers, an emergent technique for micro and nano-object manipulation, has been presented. The physical basis and the main experimental procedures we used for 1D and 2D patterning are described. In addition, our new recent work on the application of PV tweezers in various fields has been reported. Specifically, it is demonstrated that PV tweezers are a simple and flexible tool to fabricate diffractive optical components with arbitrary patterns or metallic nanostructures exhibiting plasmonic effects. Finally, it has been reported for the first time efficient trapping and patterning of various types of bio-objects (spores, pollen and its fragments). A rapid development of these and other applications of the technique is expected in the near future.

\section{Acknowledgements}

This work was supported by projects MAT2011-28379-C03 and MAT2014-57704-C3.

\section{REFERENCES}

[1] S. S. Sarkisov, M. J. Curley, N. V. Kukhtarev, A. Fields, G. Adamovsky, C. C. Smith, and L. E. Moore, "Holographic surface gratings in iron-doped lithium niobate," Appl. Phys. Lett. 79, 901 (2001).

[2] H. A. Eggert, F. Y. Kuhnert, K. Buse, J. R. Adleman, and D. Psaltis, "Trapping of dielectric particles with lightinduced space-charge fields," App. Phys. Lett. 90, 241909 (2007).

[3] X. Zhang, J. Wang, B. Tang, X. Tan, R. A. Rupp, L. Pan, Y. Kong, Q. Sun, and J. Xu, “Optical trapping and manipulation of metallic micro/nanoparticles via photorefractive crystals," Opt. Express 17, 9981 (2009).

[4] B. Sturmann, and V. Fridkin, "Photovoltaic and Photorefractive effects in noncentosymetric materials," Gordon and Breach Science publishers, Philadelphia (1992).

[5] E. de Miguel, J. Limeres, M. Carrascosa, and L. Arizmendi, "Study of developing thermal fixed holograms in lithium niobate," J. Opt. Soc. Am. B 17, 1440 (2000).

[6] M. Esseling, F. Holtmann, M. Woerdemann, and C. Denz, "Two-dimensional dielectrophoretic particle trapping in a hybrid crystal/PDMS-system," Opt. Express 18, 17404 (2010).

[7] H. Burgos, M. Jubera, J. Villarroel, A. García-Cabañes, F. Agulló-López, and M. Carrascosa, "Role of particle anisotropy and deposition method on the patterning of nano-objects by the photovoltaic effect in $\mathrm{LiNbO}_{3}$," Opt. Mat. 35, 1700 (2013).

[8] S. Glaesener, M. Esseling, and C. Denz, "Multiplexing and switching of virtual electrodes in optoelectronic tweezers based on lithium niobate," Opt. Lett. 37, 3744 (2012).

[9] M. Esseling, A. Zaltron, C. Sada, and C. Denz, "Charge sensor and particle trap based on z-cut lithium niobate," Appl. Phys. Lett. 103, 061115 (2013).

[10] J. F. Muñoz-Martínez, I. Elvira, M. Jubera, A. García-Cabañes, J. Bruno Ramiro, C. Arregui, and M. Carrascosa, "Efficient photo-induced dielectrophoretic particle trapping on $\mathrm{Fe}: \mathrm{LiNbO}_{3}$ for arbitrary two dimensional patterning," Opt. Mat. Express, accepted, in press (March, 2015).

[11] J. Villarroel, H. Burgos, A. García-Cabañes, M. Carrascosa, A. Blázquez-Castro, and F. Agulló-López, "Photovoltaic versus optical tweezers," Opt. Express 19, 24320 (2011).

[12] C. Arregui, J. B. Ramiro, A. Alcázar, A. Méndez, H. Burgos, A. García-Cabañes, and M. Carrascosa, "Optoelectronic tweezers under arbitrary illumination patterns: theoretical simulations and comparison to experiment," Opt. Express 22, 29099 (2014).

[13] J. Matarrubia, A. García-Cabañes, J. L. Plaza, F. Agulló-López, and M. Carrascosa, "Optimization of particle trapping and patterning via photovoltaic tweezers: role of light modulation and particle size,” J. Phys. D 47, 265101 (2014). 
[14] A. Blázquez-Castro, J. C. Stockert, B. López-Arias, A. Juarranz, F. Agulló-López, A. García-Cabañes, and M. Carrascosa, "Tumour cell death induced by the bulk photovoltaic effect of $\mathrm{LiNbO}_{3}: \mathrm{Fe}$ under visible light irradiation," Photochem. Photobio. Sci. 10, 956 (2011).

[15] J. B. Ramiro, A. Blázquez-Castro, A. García-Cabañes, L. Arizmendi, A. Méndez, A. Alcazar, J. C. Stockert, F. Agulló-López and M. Carrascosa, "Photvoltaic $\mathrm{LiNbO}_{3}$ particles: Applications to Biomedecine/Biophotonics," Proc. of Conference Trends in Nanotechnology (TNT), Sevilla, Spain, September (2012). 